\title{
Research at the Institute of Astronomy and Astrophysics of the Université Libre de Bruxelles*
}

\author{
Drisya Karinkuzhi, Nicolas Chamel, Stéphane Goriely, Alain Jorissen, \\ Dimitri Pourbaix, Lionel Siess, Sophie Van Eck
}

Institut d'Astronomie et d'Astrophysique, Université Libre de Bruxelles, CP 226, Boulevard du Triomphe, B-1050 Brussels, Belgium

\begin{abstract}
Over the years, a coherent research strategy has developed in the field of stellar physics at the Institute of Astronomy and Astrophysics (IAA). It involves observational studies (chemical composition of giant stars, binary properties, tomography of stellar atmospheres) that make use of the large ESO telescopes as well as of other major instruments. The presence of a high-resolution spectrograph on the 3.6-m Devasthal Optical Telescope (DOT) would therefore be highly beneficial to IAA research. These observations are complemented and supported by theoretical studies of mass transfer in binary systems, of standard and non-standard stellar evolution (including the modelling of stellar hydrodynamical nuclear burning for application to certain thermonuclear supernovae) and of nuclear astrophysics (a field in which IAA has been recognized for a long time as an international centre of excellence), including the theory of nucleosynthesis. IAA also addresses the endpoints of stellar evolution as it is carrying out research on the compact remnants of stellar evolution of massive stars: neutron stars.
\end{abstract}

\section{Nuclear astrophysics and nucleosynthesis}

One of the major issues in modern astrophysics concerns the analysis and understanding of the present composition of the Universe and its various constituting objects. Nucleosynthesis models aim at explaining the origin of the different nuclei observed in nature by identifying the possible processes able to synthesize them. Though the origin of most of the nuclides lighter than iron through the various hydrostatic and explosive burning stages in stars is now quite well understood, the synthesis of the heavy elements (i.e. heavier than iron) through the so-called slow neutron-capture process (sprocess), rapid neutron-capture process (r-process), and the photodisintegration process (p-process) remains obscure in many respects (see Fig. 1).

The stellar nucleosynthesis requires a detailed knowledge not only of the astrophysical sites and physical conditions in which the processes take place, but also the nuclear structure and interaction properties for all the nuclei involved. For that purpose, IAA has developed over the years an extended library of nuclear data of astrophysical interest called BRUSLIB (BRUSsels LIBrary). BRUSLIB

\footnotetext{
${ }^{*}$ Institut d'Astronomie et d'Astrophysique, ULB
} 


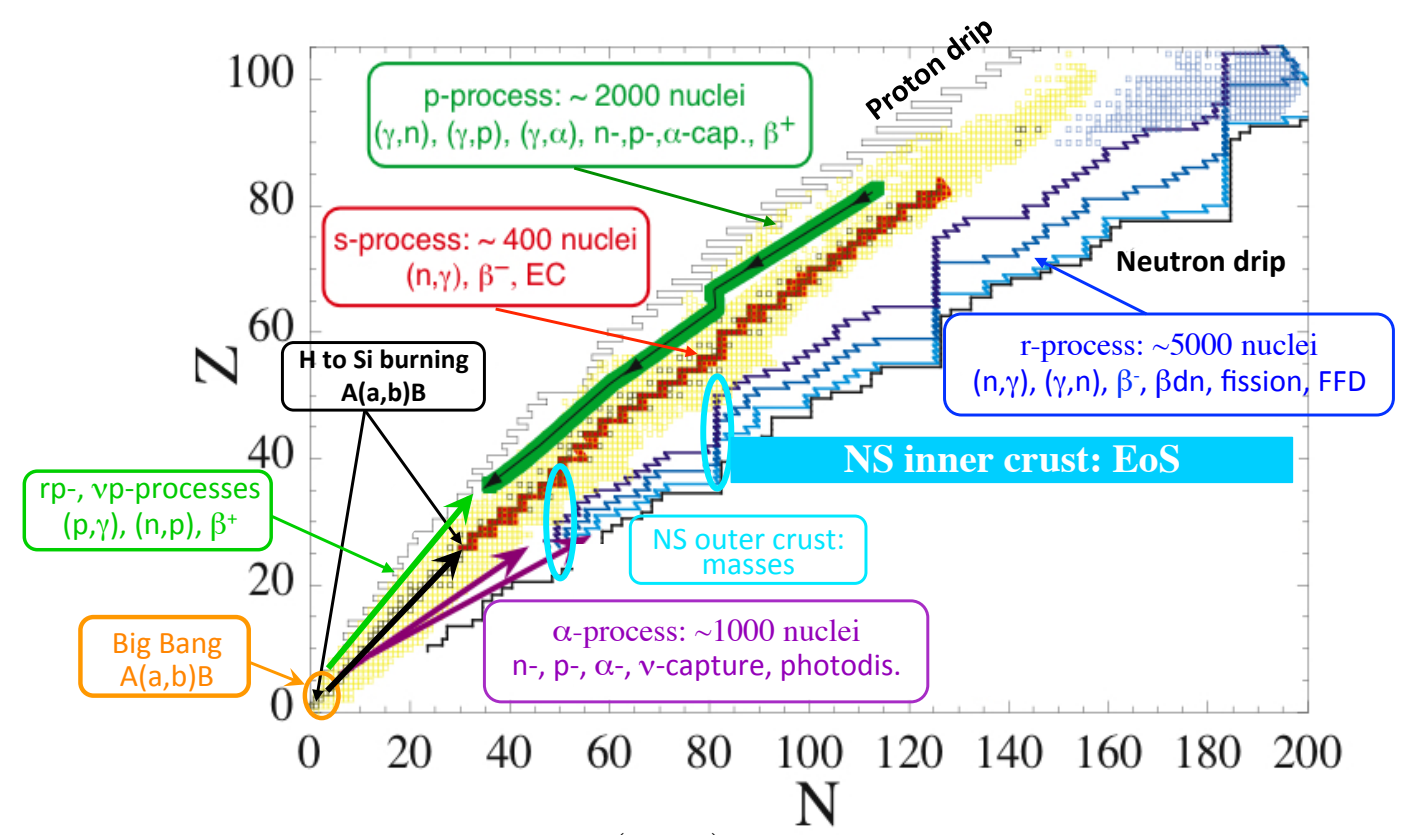

Figure 1: Schematic representation in the $(N, Z)$ plane of the different low-energy nuclear astrophysics processes. For each process, the nuclear needs are sketched. The open black squares correspond to stable or long-lived nuclei, the yellow squares to the nuclei for which masses have been measured and the blue squares to those which may fission.

provides various thermonuclear reaction rates (nucleon and alpha particle captures, as well as photodisintegrations) and the necessary ingredients like masses, level densities, $\gamma$-ray strength functions and fission barriers to calculate the reaction rates (Xu et al. 2013).

\section{Compact stars}

The study of neutron stars represents another important research topic at the IAA. Formed in gravitational core-collapse supernova explosions of massive stars, neutron stars contain matter at densities exceeding that found inside the heaviest atomic nuclei, and are endowed with the strongest magnetic fields known, which could reach millions of billions times that of the Earth. Neutron-star observations thus offer the unique opportunity to explore the properties of matter under extreme conditions, which cannot be reproduced in the laboratory.

Over the past years, we have been developing self-consistent nuclear models for describing such environments. In particular, we have calculated a series of unified equations of state spanning the current lack of knowledge of dense matter, and treating consistently all regions of a neutron star, from their solid crust to their liquid core. We have also been exploring the observational signatures of nuclear superfluidity in neutron-star interiors, especially in relation to pulsar sudden spin-ups.

\section{Stellar evolution of single and binary stars}

Another major theme of research at IAA is the study of stellar evolution. The STAREVOL code is a recognized key player in the stellar-evolution community for its contributions in the domains of pre-main sequence evolution, angular momentum in low-mass stars, the structure of evolved stars 

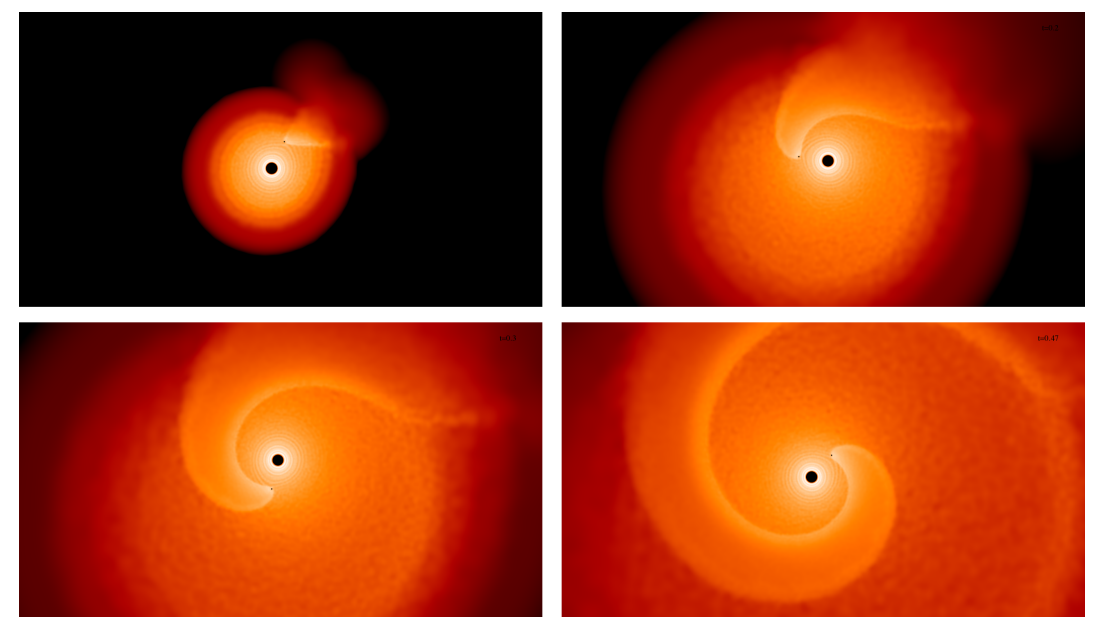

Figure 2: Evolution of the density profile in a binary system composed of a $1.2 M_{\odot}$ AGB star and a $0.6 M_{\odot}$ companion at 4 different epochs. The period of the system is 67 years.

like Asymptotic Giant Branch (AGB) stars and super-AGBs, as well as the associated s-process nucleosynthesis. STAREVOL allows the modelling of stars over a wide range of masses, chemical compositions and evolutionary stages and has been used in various contexts to study for example the role of overshooting and rotational mixing on the s-process nucleosynthesis, the swallowing of planets by giant stars, the evolution of the first stars or the accretion on young stellar objects. This code includes up-to-date nuclear reaction rates (Sect. 1) which serve for the interpretation of the abundances of AGB and post-AGB stars (Sect. 4).

The binary stellar evolution code BINSTAR (Siess et al. 2013) is also developed at IAA and allows the simultaneous computation of the evolution of the two stars in a binary system along with that of the orbital elements (separation and eccentricity). The BINSTAR code has been used to investigate episodic mass transfer at periastron in eccentric systems, the evolution of short-period systems such as Algols or to explain the high eccentricity of peculiar long-period systems hosting a helium white dwarf. It is currently upgraded to account for the generation of eccentric orbits due to the presence of a circumbinary disk which seems to be necessary to understand the orbital properties (Sect. 4) of low- and intermediate-mass long-period binaries such as Ba dwarf, Ba or $\mathrm{CH}$ stars.

In parallel, we are developing a new module in the 3D smooth particle hydrodynamic code PHANTOM (Price et al. 2017) to simulate the dust-driven wind and wind mass transfer in a binary system containing an AGB star and a lower mass companion. The results of these simulations (Fig. 2) will provide more realistic estimates of the mass and angular-momentum transfer rates that will feed back in the BINSTAR code. These calculations will also help understanding the formation of circumbinary disks.

\section{Stellar atmospheres, abundances and binarity}

IAA has acquired a recognized expertise in the detection of spectroscopic binaries and in the analysis of their orbital elements, providing useful constraints to mass-transfer modelling (Sect. 3).

Abundance determinations of stars exhibiting anomalous abundance patterns, either due to an internal nucleosynthesis (Asymptotic Giant Branch stars of M, S and C spectral types) or to an external pollution (barium, $\mathrm{CH}$, CEMP stars) are routinely performed thanks to high-resolution spectra obtained for example at ESO or from the HERMES spectrograph mounted on the 1.2-m MERCATOR telescope at La Palma (Canary Island) and owned by KULeuven (Raskin et al. 2011). MARCS model atmosphere computations and spectral synthesis with the TURBOSPECTRUM code are used to derive abundance profiles, which are then compared to the stellar evolution and nucleosynthetic predictions (Fig. 3; Sections 1 and 3). A good example of a successful confrontation between theory 


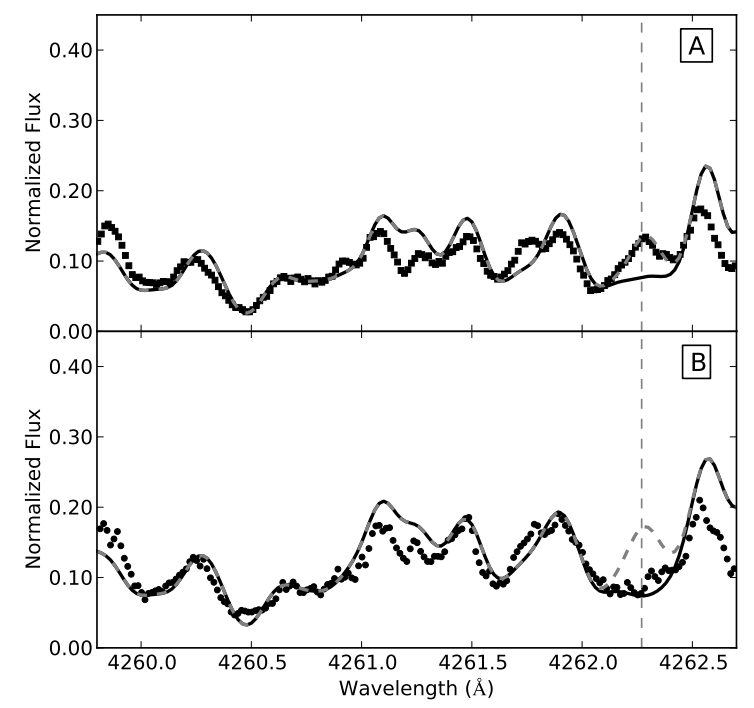

Figure 3: Observed and synthetic spectra around the Tc I $4262.270 \AA$ line of the extrinsic, Tc-poor S star BD Cam (panel A) and the intrinsic, Tc-rich S star o1 Ori (panel B). Dots represent high-resolution HERMES spectra; synthesis with no Tc (dashed line) and enhanced Tc (continuous line) abundances are plotted.

and observations is provided by a recent study of the IAA group (Neyskens et al. 2015) where abundances of key elements like $\mathrm{Tc}, \mathrm{Nb}$, and $\mathrm{Zr}$ in s-process-enriched stars gave access to the s-process operation temperature and to the duration of the AGB phase. Another research theme concerns the atmospheres of evolved giants, investigated either using interferometry, or spectroscopic tomography, to probe their surface structure and atmospheric velocity fields.

\section{Binaries from space}

For almost 30 years, Europe has been the leader in space astrometry, initially with Hipparcos (ESA 1997) and now with Gaia (Gaia Collaboration, 2016). IAA holds a key position in the processing of the Gaia astrometric signal of binaries. Such a specific treatment is crucial as the binary nature of the source could affect the parallax and proper motion derived assuming the default single star model. Using the appropriate models also gives access to parameters that would remain hidden otherwise (e.g. orbital period, eccentricity, ...). In the near future, Gaia will therefore supply the other IAA teams with a revised binary distribution in the Galaxy (a few millions).

Besides the positions, Gaia also repeatedly measures the instantaneous brightness of the sources, thus allowing the detection of eclipsing binaries and, possibly, the modelling of their light curve. The latter is precisely the task of some members of the IAA Gaia team. Once again, the resulting orbits will complement the astrometric binary census as the range of orbital periods for eclipsing and astrometric binaries seldom overlap.

\section{References}

Chamel N. 2013, Physical Review Letters, 110, 011101

ESA 1997, The Hipparcos and Tycho Catalogues, ESA SP, 1200

Gaia Collaboration et al. 2016, A\&A, 595, A2

Jorissen A., Van Eck S., Van Winckel H. et al. 2016, A\&A, 586, 158

Neyskens P., Van Eck S., Jorissen A. et al. 2015, Nature 517, 174

Pourbaix D., Boffin H. 2016, A\&A, 586, A90

Price F. J., Wurster J., Niwon C. et al. 2017, arXiv170203930

Raskin G., Van Winckel H., Hensberge H. et al. 2011, A\&A 526, A69

Siess L., Izzard R. G., Davis P. J., Deschamps R. 2013, A\&A 550, A100

Van Eck S., Goriely S., Jorissen A., Plez, B. 2001, Nature 412, 793

Xu Y., Goriely S., Jorissen A., Chen G. L., Arnould M. 2013, A\&A, 549, 106 